\title{
TIGHT RIESZ GROUPS
}

\author{
R. J. LOY and J. B. MILLER
}

(Received 5 August 1969, revised 8 December 1969)

Communicated by J. P. O. Silberstein

\section{Introduction}

The theory of partially ordered topological groups has received little attention in the literature, despite the accessibility and importance in analysis of the group $R^{m}$. One obstacle in the way of a general theory seems to be, that a convenient association between the ordering and the topology suggests that the cone of all strictly positive elements be open, i.e. that the topology be at least as strong as the open-interval topology $\mathfrak{U}$; but if the ordering is a lattice ordering and not a full ordering then $\mathfrak{U}$ itself is already discrete. So to obtain in this context something more interesting topologically than the discrete topology and orderwise than the full order, one must forego orderings which make lattice-ordered groups: in fact, the partially ordered group must be an antilattice, that is, must admit no nontrivial meets or joins $\left(s \in e ~ \S 2,10^{\circ}\right)$.

There is of course the order topology; for this however it is necessary to lay down rather complicated conditions at the outset, to ensure that the group is a topological group.

In this paper we develop the theory of a class of commutative groups for which these difficulties can to some extent be overcome, though no new locally compact groups are found in the class. The theory is based on a strengthened form of the well-known interpolation property due to F. Riesz (1940); we call it here the 'tight Riesz property'. We are in the first instance concerned with tight Riesz groups $(G, \leqq, \mathfrak{U})$ without pseudozeros. For such a group the closure $\bar{P}$ under $\mathfrak{U}$ of the positive cone of the ordering $\leqq$ is itself the positive cone of an ordering $\leqslant$. The group $(G, \leqq, \mathfrak{u})$ is order-dense and not discrete, and although it is therefore an antilattice, $(G, \preccurlyeq ; \mathfrak{H})$ is a closely related partially ordered topological group which may very well be lattice-ordered; so that the lack of a lattice structure in $(G, \leqq)$ need not be an inconvenience. When $(G, \leqq)$ is Archimedean ordered, $(G, \mathfrak{U})$ has no compact subgroups other than $(0)$, and so is radical-free.

The example afforded by $R^{m}$, the additive group of real $m$-tuples, uses the orderings $\leqq$ and $\leqslant$ defined by:

$$
\left\langle\alpha_{1}, \alpha_{2}, \cdots, \alpha_{m}\right\rangle>0 \text { iff } \alpha_{1}>0, \alpha_{2}>0, \cdots, \alpha_{m}>0,
$$




$$
\left\langle\alpha_{1}, \alpha_{2}, \cdots, \alpha_{m}\right\rangle \succcurlyeq 0 \text { iff } \alpha_{1} \geqq 0, \alpha_{2} \geqq 0, \cdots, \alpha_{m} \geqq 0 .
$$

Of these the second, though it seems the more frequently used by analysts and algebraists alike, has the disadvantage from which the first is free, of not giving special status to the elements for which one or more of the $\alpha$ 's vanish, the remaining being positive. The first classifies them immediately as not positive but pseudopositive. This is a case of the general result $\left(\S 2,6^{\circ}\right)$ that $\partial P$, the boundary of the positive cone, consists precisely of 0 and the pseudopositive elements, so that one passes from $\leqq$ to $\leqslant$ by reclassifying the pseudopositive elements as positive.

The cone of strictly positive elements in $\left(R^{m}, \leqq\right)$ is open in the Euclidean topology $\mathbb{E}$, which coincides with $\mathfrak{U}$. The extraction of the Euclidean topology from the $\preccurlyeq$ order structure is less straightforward. $\left(R^{m}, \leqq, \mathfrak{u}\right)$ is a tight Riesz group without pseudozeros, Archimedean ordered, and locally compact. Indeed, we show that, up to isomorphism, these are the only such groups $\left(\S 5,2^{\circ}\right)$.

Contents. $\S 2$. A summary of the basic theory of partially ordered topological groups, the Riesz interpolation properties and some compactness results.

$\S 3$. Upper and lower bounds. The condition $[*]$, and the consequence that $(G, \preccurlyeq)$ is lattice-ordered.

$\S 4$. Consequences of the lattice structure of $(G, \preccurlyeq)$ when $(G, \leqq)$ has no pseudozeros. A lemma concerning the existence of small positive elements.

$\S 5$. The full implication of $\left[{ }^{*}\right]$ : the complete isomorphism for some $m$ of $(G, \leqq, \leqslant, \mathfrak{U})$ and $\left(R^{m}, \leqq, \leqslant,(\mathfrak{E})\right.$.

$\S 6$. Some examples.

For convenience, $\S 2$ repeats a portion from the second author's previous paper (1970). The present paper is a revised form of an unpublished paper by the second author. The first author has supplied the results in $\S 5$, and this section now replaces an earlier unsatisfactory discussion of conditions for the divisibility of $G$ and its consequences. We are grateful for comments by Diana Frost and Ian Wright, and the referee, which have also led to improvements. Generally, we owe much to the basic studies of Riesz groups by L. Fuchs. The dense antilattices of Fuchs (1965), pp 20-21, are tight Riesz groups in our terminology. For an application of tight Riesz groups to a generalization of the Stone-Weierstrass theorem see Miller (1972).

\section{Partially ordered topological groups}

We confine attention throughout this paper to Abelian groups, which we write additively. Thus by 'group' we always mean 'Abelian group'.

A partially ordered group $(G, \leqq)$ is a group $G$ which is a partially ordered set under $\leqq$ and is such that for all $a, b, c \in G$, if $a<b$ then $a+c<b+c$ (so that also $-b<-a$ ). We write

$$
P=\{a \in G: a \geqq 0\}, \quad P^{*}=P \backslash\{0\},
$$


and call $P$ the positive cone of the ordering. It satisfies

$$
P+P \subseteq P, \quad P \cap(-P)=\{0\} ;
$$

and conversely any subset $P$ with these properties is the positive cone of some partial ordering $\leqq$ making $(G, \leqq)$ a partially ordered group. The ordering is full if and only if $P \cup(-P)=G$. We assume in general that the ordering is not trivial, i.e. that $P^{*} \neq \emptyset$. It follows that $G$ is infinite. $G$ need not be torsion-free, but there can be no torsion elements in $P^{*}$. For some of the properties of partially ordered groups given below, see L. Fuchs (1963).

We say that $(G, \leqq)$ is directed to the right when to any pair $a, b \in G$ there exists some $c \in G$ for which $a \leqq c, b \leqq c$. This implies the existence of $c^{\prime} \in G$ for which $a<c^{\prime}, b<c^{\prime}$. There is a similar definition for directed to the left. Since $G$ is a group, each implies the other, so it suffices to say merely that the group is directed.

Henceforth suppose that $(G, \leqq)$ is a partially ordered group.

$1^{\circ}$. The following assertions are equivalent: (i) $G$ is directed, (ii) $P$ generates $G$, (iii) $G=P-P$.

Call $(G, \leqq)$ isolated when $n a>0$ for some positive integer $n$ implies $a>0$.

We say $(G, \leqq)$ is order-dense when for every pair $a, b \in G$ such that $a<b$ there exists at least one $c \in G$ such that $a<c<b$. An element $x$ is an atom of $G$ if $x>0$, and $0<a<x$ for no $a \in G$. Clearly, $(G, \leqq)$ is order-dense if and only if there exist no atoms in $G$.

We use the following notation for order intervals in $G$ :

$$
\begin{aligned}
& (a, b)=\{x: a<x<b\},[a, b]=\{x: a \leqq x \leqq b\}, \\
& (a, \infty)=\{x: a<x\},(-\infty, b)=\{x: x<b\},
\end{aligned}
$$

with the obvious variants for half-open intervals.

The open-interval topology $\mathfrak{U}$ is the topology generated on the set $G$ by taking the collection of all sets of the form $(a, \infty),(-\infty, b)$ as a subbase. It is easily shown that the collection of all sets of the form $(a, b)$ then also forms a subbase for $\mathfrak{U}$, though perhaps not a base.

An element $w \in G$ is called pseudopositive when $w \geqq 0$ but $a>0$ implies $a+w>0$, that is

$$
w+P^{*} \subseteq P^{*}, w \geqq 0 .
$$

An element $\theta$ is a pseudozero if $\theta$ and $-\theta$ are both pseudopositive, that is

$$
\theta+P^{*}=P^{*}, \theta \neq 0 .
$$

$2^{\circ}$. The topological space $(G, \mathfrak{u})$ is a $T_{1}$-space if and only if $(G, \leqq)$ has no pseudozeros.

We recall that if $\mathfrak{T}$ is some topology on $G,(G, \mathfrak{T})$ is called a topological group if the maps $\langle a, b\rangle \mapsto a+b$ of $G \times G$ onto $G$, and $a \mapsto-$ a of $G$ onto $G$, are conti- 
nuous. For the properties of topological groups we refer to E. Hewitt and K. A. Ross (1963).

$3^{\circ}$. ThEOREM. If $(G, \leqq)$ is order-dense then $(G, \mathfrak{U})$ is a topological group and a regular topological space; and $\mathfrak{U}$ is the weakest topology $\mathfrak{T}$ such that $(G, \mathfrak{T})$ is a topological group in which $P^{*}$ is open.

If further $(G, \leqq)$ has no pseudozeros, then the topology $\mathfrak{U}$ is Hausdorff and completely regular.

Proof. Given $s, t \in G$ and a subbase neighbourhood $U=(a, b)$ of $s+t$, we deduce from the fact that $G$ is order-dense that there exist $x, y$ such that

$$
a-t<x<s<y<b-t .
$$

Then $V=(x, y)$ and $W=(a-x, b-y)$ are neighbourhoods of $s$ and $t$ respectively such that $V+W \subseteq U$. By taking a finite collection of subbase sets like $U$ and forming intersections, we get the same result for an arbitrary base neighbourhood of $s+t$, showing that addition is continuous. That inversion is continuous follows trivially from the nature of the topology. So $(G, \mathfrak{u})$ is a topological group. For a proof that it is regular see, for example, Hewitt and Ross (1963), p. 19.

By definition of $\mathfrak{u}, P^{*}$ is open in $(G, \mathfrak{u})$. Conversely, if $(G, \mathfrak{I})$ is a topological group in which $P^{*}$ is open, then $a+P^{*}, b-P^{*}$ are open for all $a, b \in G$, i.e. I contains the subbase of $\mathfrak{U}$, and therefore $\mathfrak{I} \supseteq \mathfrak{U}$.

If $G$ has no pseudozeros then $\mathfrak{U}$ is $T_{1}$, by $2^{\circ}$. Regularity and the $T_{1}$ property imply that $\mathfrak{U}$ is Hausdorff; and completely regular; see Hewitt and Ross (1963), p. $70 . / 1$

Next we introduce the Riesz interpolation properties. These are meaningful when $(G, \leqq)$ is any partially ordered set, but we continue to suppose that $(G, \leqq)$ is a partially ordered group. $(G, \leqq)$ is said to have the tight Riesz property if, for every four (not necessarily distinct) elements $a_{1}, a_{2}, b_{1}, b_{2}$ in $G$ such that

$$
a_{i}<b_{j}
$$

for $i, j=1,2$ there exists $c \in G$ such that

$$
a_{i}<c<b_{j}
$$

for $i, j=1,2 .{ }^{1}$ If $<$ is replaced by $\leqq$ at all places in (2.4) and (2.5), we get instead the definition of the loose Riesz property.

A tight Riesz group is a partially ordered group which is directed and has the tight Riesz property. Loose Riesz groups are defined analogously.

The loose Riesz property is the form of interpolation condition originally introduced by F. Riesz (1940), and the group-theoretic consequences are described in detail in Fuchs (1965). Our main concern here is with the tight property, and

1 We sometimes abbreviate (2.4) to $a_{1}, a_{2}<b_{1}, b_{2}$; and similarly for other inequalities. 
we shall say little more about loose Riesz groups beyond using some characterizations of them which, by obvious modifications, give characterizations of tight Riesz groups.

$4^{\circ}$. THEOREM. Let $(G, \leqq)$ be a directed partially ordered group. Each of the following conditions implies the others.

I. $(G, \leqq)$ is a tight Riesz group.

II. For any two positive integers $p, q$, if elements $a_{1}, a_{2}, \cdots, a_{p}$ and $b_{1}$, $b_{2}, \cdots, b_{q}$ satisfy

$$
a_{i}<b_{j} \quad(i=1,2, \cdots, p ; j=1,2, \cdots, q)
$$

then there exists an element $c$ satisfying

$$
a_{i}<c<b_{j} \quad(i=1,2, \cdots, p ; j=1,2, \cdots, q) .
$$

III. Given any $r>1$, for all $a_{1}, a_{2}, \cdots, a_{r}$ in $P^{*}$,

$$
\left(0, a_{1}\right)+\left(0, a_{2}\right)+\cdots+\left(0, a_{r}\right)=\left(0, a_{1}+a_{2}+\cdots+a_{r}\right) .
$$

JV. Given $m+n$ elements $a_{1}, a_{2}, \cdots, a_{m}$ and $b_{1}, b_{2}, \cdots, b_{n}$, all in $P^{*}$ and for which

$$
a_{1}+a_{2}+\cdots+a_{m}=b_{1}+b_{2}+\cdots+b_{n},
$$

there exist $m n$ elements $c_{i j}$, all in $P^{*}$, such that

$$
\begin{array}{ll}
a_{i}=c_{i 1}+c_{i 2}+\cdots+c_{i n} & (i=1,2, \cdots, m), \\
b_{j}=c_{1 j}+c_{2 j}+\cdots+c_{m j} & (j=1,2, \cdots, n) .
\end{array}
$$

V. Given elements $a, b_{1}, b_{2}, \cdots, b_{k}$ in $P^{*}$ such that

$$
a<b_{1}+b_{2}+\cdots+b_{k},
$$

there exist elements $a_{1}, a_{2}, \cdots a_{k}$ in $P^{*}$ such that

$$
a=a_{1}+a_{2}+\cdots+a_{k} ; a_{i}<b_{i} \quad(i=1,2, \cdots, k) .
$$

Proof. The equivalence of analogous statements got by replacing 'tight' by 'loose' in $\mathrm{I}$, and $P^{*}$ by $P,(0, a)$ by $[0, a],<$ by $\leqq$ throughout, is proved in Fuchs (1965), § 2; the proof of the present theorem is obtained by making the obvious modifications in this proof of the loose case. //

$5^{\circ}$. THEOREM. A tight Riesz group $(G, \leqq)$ is order-dense, and a topological group under its open-interval topology $\mathfrak{U} . \mathfrak{U}$ is not discrete, and the collection of all open order-intervals $(a, b)$ forms a base for $\mathfrak{H}$.

Proof. The tight Riesz property implies immediately that $(G, \leqq)$ is orderdense, so $(G, \mathfrak{u})$ is a regular topological group, by $3^{\circ}$. If $V=\bigcap_{i=1}^{n}\left(a_{i}, b_{i}\right)$ is a base neighbourhood of $c$, there exist $a, b \in G$ such that 


$$
a_{i}<a<c<b<b_{j} \quad(i, j=1,2, \cdots, n),
$$

by $4^{\circ}$. Thus the sets $(a, b)$ form a base for $\mathfrak{U}$. Further, any neighbourhood of $c$ contains elements other than $c$, so $\{c\}$ is not open. Since the topology of a topological group is translation-invariant, $\mathfrak{H}$ is not discrete. //

We shall make repeated use of this theorem.

$6^{\circ}$. In a tight Riesz group $(G, \leqq, \mathfrak{H})$ :

(i) $\bar{P}=\overline{P^{*}}, \partial P=\partial\left(P^{*}\right), 0 \in \partial\left(P^{*}\right),(\bar{P})^{\circ}=P^{*}, \overline{G \backslash P}=\overline{G \backslash \bar{P}}, \partial P=\hat{\partial} \bar{P}$; where ${ }^{-}, \partial$ and ${ }^{\circ}$ denote topological closure, boundary and interior respectively;

(ii) $\partial P$ consists of all the pseudopositive elements together with 0 ;

(iii) $\bar{P} \cap(-\bar{P})$ consists of all pseudozeros together with 0 , and equals $\partial P \cap(-\partial P)$.

Proof. (i) Clearly $\bar{P} \supseteq \overline{P^{*}}$. If $x \in \bar{P}$ then every base neighbourhood $U=$ $(x-a, x+a), a>0$, meets $P$. Suppose $s \in U \cap P$; if $s \neq 0$ then $U$ meets $P^{*}$, while if $s=0$ then $x-a<0<x+a$ so there exists $t \in G, x-a<0<t<x+a$, and again $U$ meets $P^{*}$. Thus $x \in \overline{P^{*}}$. This proves the first equation, and the others follow easily, or are proved by similar arguments.

(ii) Let $w$ be pseudopositive or zero. If $a>0$ take $0<b<a$; then $w+b>0$ so $(w-a, w+a)$ meets $P^{*}$ in $w+b$. Such intervals form a base in $\mathfrak{U}$ at $w$ as we have seen; therefore $w \in \partial\left(P^{*}\right)=\partial P$.

Conversely if $w_{1} \in \partial P$ then $a>0$ implies $w_{1}-a<c<w_{1}+a$ for some $c \geqq 0$ and hence $a+w_{1}>0$. So $w_{1}$ is pseudopositive or zero or positive; and $w_{1}$ cannot be positive, otherwise $w_{1} \in P^{*}$, and $P^{*}$ is disjoint from $\partial P^{*} \equiv \partial P$.

(iii) Clearly $0 \in \bar{P} \cap(-\bar{P})$. Suppose $s \in \bar{P} \cap(-\bar{P}), s \neq 0$. We cannot have $s \in P \cap(-P)$, by (2.1). If $s \in P \cap(-\partial P)$ then $(-s-e,-s+e)$ meets $P$, so $s<e$ for every $e>0$, which contradicts order-denseness since $s>0$; thus

$$
s \notin P \cap(-\partial P) .
$$

Likewise $s \notin \partial P \cap(-P)$. There remains the possibility $s \in \partial P \cap(-\partial P)$; but by (ii) this implies that $s$ is a pseudozero.

Conversely if $\theta$ is a pseudozero then $\theta \in \partial P \cap(-\partial P)$ by (ii), so $\theta \in \bar{P} \cap(-\bar{P})$.

For any subsemigroup $P$ of a topological group, $\bar{P}+\bar{P} \subseteq \bar{P}$; so

$7^{\circ}$. In a tight Riesz group $(G, \leqq, \mathfrak{U}), \bar{P}$ is a positive cone if and only if $(G, \leqq)$ has no pseudozeros.

When $\bar{P}$ is a positive cone, we will write the corresponding ordering as $\leqslant$. Thus $a \succ 0$ in a tight Riesz group without pseudozeros asserts that $a>0$ or $a$ is pseudopositive; and

$$
a \geqslant 0 \text { if and only if } x>0 \Rightarrow x+a>0 .
$$


Writing $\llbracket a, b \rrbracket=\{x \in G: a \leqslant x \leqslant b\}$ for the corresponding closed order-interval, we have

$8^{\circ}$. In a tight Riesz group without pseudozeros, if $a<b$ then

$$
[a, b] \subseteq(a, b)^{-}=\llbracket a, b \rrbracket,
$$

and $\llbracket a, b \rrbracket$ is a closed set.

The proof is left to the reader.

Cases when $\mathfrak{U}$ is discrete. We have remarked that the tight Riesz property prevents $(G, \mathfrak{U})$ being discrete, and now look briefly at neighbouring cases. For a partially ordered group ( $G, \leqq)$, let $a \wedge b$ and $a \vee b$ have their usual meanings of meet and join. Call $(G, \leqq)$ an antilattice if, for a given pair $a, b \in G, a \wedge b$ exists only when $a \wedge b$ equals $a$ or $b$, i.e. only the trivial meets exist. It can be shown that $(G, \leqq)$ is an antilattice if and only if only the trivial joins exist.

A necessary and sufficient condition for $(G, \leqq)$ to be an antilattice is: for all $a, b \in G$,

$$
a \wedge b=0 \text { implies } a=0 \text { or } b=0 .
$$

For a proof see Fuchs (1965), p. 19. (Note that the term 'antilattice' there is confined to loose Riesz groups.) It follows immediately from the definitions that

$9^{\circ}$. A tight Riesz group is an antilattice.

$10^{\circ}$. If $(G, \mathfrak{u})$ is a topological group but not an antilattice, then $\mathfrak{U}$ is discrete.

ProOF. By (2.8) there exist $a>0, b>0$ with $a \wedge b=0$. But then $(-a, a) \cap$ $(-b, b)$ is an open set containing only 0 . Thus every singleton is open, and $\mathfrak{U}$ is discrete. //

For a partially ordered set $(G, \leqq)$ any two of the following implies the third: (i) $(G, \leqq)$ is lattice-ordered; (ii) $(G, \leqq)$ is fully ordered; (iii) $(G, \leqq)$ is an antilattice with respect to both $\wedge$ and $\vee$. Therefore

$11^{\circ}$. If $(G, \mathfrak{u})$ is a topological group, and $(G, \leqq)$ is lattice-ordered but not fully ordered, then $\mathfrak{U}$ is discrete,

Compactness. We pass on to some results about compactness and local compactness, first noting

$12^{\circ} .(G, \mathfrak{H})$, if a topological group, is not compact.

Proof. Take $a>0$ and write $U=(-a, a)$. Then $t+U$ is a neighbourhood of $t$, so if $(G, \mathfrak{H})$ is compact there exists a subset $\left\{t_{1}, t_{2}, \cdots, t_{n}\right\}$ of $G$ such that $\left\{t_{j}+U: j=1,2, \cdots, n\right\}$ covers $G$. That is, for every $x \in G$ there exists some integer $j_{x}, 1 \leqq j_{x} \leqq n$, such that

$$
x-a<t_{j_{x}}<x+a .
$$


Taking $x=a, 3 a, 5 a, \cdots$ gives an infinite set of pairwise disjoint subsets of $G$, each containing some $t_{j}$, which is impossible. //

A similar argument can be applied to compact subgroups of $G$, to show that any such subgroup $H$ meets $P$ only in $\{0\}$. (The choice $x=a$ in the above proof is possible only if $H \cap P \neq\{0\}$, so the proof fails to dispose of all compact subgroups.) Later, in $\S 4,7^{\circ}$, we adapt the argument to show that $G$ contains no compact subgroups other than (0), at least for the class of tight Riesz groups considered in $\S 4$ and subsequently.

If for some $a>0$ the closure $(0, a)^{-}$is non-empty and compact, then $(G, \mathfrak{H})$ is locally compact; for the $(0, a)$ is non-empty, so there exists some $b$ such that $(-b, a-b)$ is a neighbourhood of 0 , and then for any $x \in G,(x-b, x+a-b)$ is a neighbourhood of $x$ with compact closure. The converse is also true:

$13^{\circ}$. A tight Riesz group $(G, \leqq, \mathfrak{U})$ is locally compact if and only if there exists $a>0$ such that $(0, a)$ has compact closure.

ProOf. It remains to verify necessity. If $V$ is a neighbourhood of 0 then there exist $a, b$ with $0 \in(a, b) \subseteq V$. If also $\bar{V}$ is compact then $(a, b)^{-}$is compact and so therefore is $(0, b-a)^{-}$. /I

To improve upon this result, we introduce a further axiom. $(G, \leqq)$ is called Archimedean ordered when $a>0, b>0$ implies $n a>b$ for some positive integer $n$. An equivalent formulation is: for every $a>0$,

$$
P^{*}=\bigcup_{n=1}^{\infty}(0, n a) \text {. }
$$

Among possible Archimedean-type properties on a partially ordered group, this is a rather strong one. See Fuchs (1963), p.12.

$14^{\circ}$. Let $(G, \leqq, \mathfrak{U})$ be a tight Riesz group without pseudozeros, and let $a, b \in P^{*}$, and let $(0, a)^{-}$and $(0, b)^{-}$be compact. Then

$$
(0, a)^{-}+(0, b)^{-}=(0, a+b)^{-},
$$

and $(0, a+b)^{-}$is also compact.

Proof. For any partially ordered group $G$,

$$
(0, a)+(0, b) \subseteq(0, a+b)
$$

and if $G$ is a topological group it can be shown without difficulty that

$$
(0, a)^{-}+(0, b)^{-} \subseteq(0, a+b)^{-} .
$$

For a tight Riesz group we have also

$$
(0, a)^{-}+(0, b)^{-} \supseteq(0, a+b),
$$

from $4^{\circ}$, III. In any topological group the sum of two compact subsets is compact, 
so the lefthand side of $(2.10)$ is compact and, since $\mathfrak{U}$ is Hausdorff by $3^{\circ}$, closed. Therefore $(0, a)^{-}+(0, b)^{-} \supseteq(0, a+b)^{-}$, and the result follows. //

$15^{\circ}$. Theorem. Let $(G, \leqq, \mathfrak{u})$ be a tight Riesz group, without pseudozeros and Archimedean ordered. Then $(G, \mathfrak{U})$ is locally compact if and only if $(0, a)^{-}$is compact for every $a>0$.

Proof. If $(0, a)^{-}$is compact for even one $a>0$ we know from $13^{\circ}$ that $(G, \mathfrak{U})$ is locally compact. Suppose conversely that $(G, \mathfrak{U})$ is locally compact. By $13^{\circ}$ there exists $a_{0}>0$ for which $\left(0, a_{0}\right)^{-}$is non-empty and compact. Let $a>0$. By the Archimedean property there exists a positive integer $n$ such that $(0, a) \subseteq$ $\left(0, n a_{0}\right)$. By $14^{\circ},\left(0, n a_{0}\right)^{-}$is compact. Thus $(0, a)^{-}$is compact. //

\section{Infima and suprema}

For any subset $A$ in a partially ordered group $(G, \leqq)$ write

$$
\operatorname{slb}(A)=\{x: x<a \text { for all } a \in A\}
$$

for the set of all strict lower bounds of $A$, and likewise

$$
\operatorname{sub}(A)=\{x: x>a \text { for all } a \in A\}
$$

for the set of all strict upper bounds.

For a subset $B \subseteq G$ we say that $B$ is strictly directed to the right under $<$ if for any pair of elements $b_{1}, b_{2}$ in $B$, not necessarily distinct, there exists $b_{3} \in B$ such that $b_{1}, b_{2}<b_{3}$.

We start with the following lemma, which is basic for many later results.

$1^{\circ}$. Lemma. Let $(G, \leqq, \mathfrak{u})$ be a tight Riesz group without pseudozeros; let $F$ be a non-empty compact subset of $G$. Then the set $\mathrm{slb}(F)$ of all strict lower bounds of $F$ is non-empty, and is strictly directed to the right under $<$.

Likewise sub $(F)$ is non-empty and strictly directed to the left under $<$.

PrOOF. Since the sets $(a-e, a+e)$ for $a \in F$ cover $F$ when $e>0$, there exist $a_{1}, a_{2}, \ldots, a_{l}$ such that $F \subset \bigcup_{j=1}^{l}\left(a_{j}-e, a_{j}+e\right)$. Since $(G, \leqq)$ is directed there exists $b<a_{j}-e, j=1,2, \cdots, l$; this $b \in \operatorname{slb}(F)$, so $\operatorname{slb}(F) \neq \emptyset$.

For any $s \in G, s+P^{*}$ is open. Let $x_{1}, x_{2} \in \operatorname{slb}(F)$ and form

$$
C=\bigcup_{s>x_{1}, x_{2}}\left(s+P^{*}\right) \text {. }
$$

If $a \in F$, by the tight Riesz property there exists $s$ for which $x_{1}, x_{2}<s<a$, so $a \in C ; F \subseteq C$. Since $F$ is compact there exist $s_{1}, s_{2}, \cdots, s_{k}$, all $>x_{1}, x_{2}$, such that

$$
F \subset \bigcup_{j=1}^{k}\left(s_{j}+P^{*}\right)
$$


We can find $x$ such that $x_{1}, x_{2}<x<s_{1}, s_{2}, \cdots, s_{k}$, and then $F \subset x+P^{*}$; that is, $x \in \operatorname{slb}(F)$ and $x>x_{1}, x_{2}$.

Proofs for $\operatorname{sub}(F)$ are analogous. //

One can show by examples that if $F$ is not compact $\mathrm{slb}(F)$ may not be directed.

We now proceed to consider groups of the following type:

$\left[{ }^{*}\right](G, \leqq, \mathfrak{U})$ denotes a tight Riesz group, without pseudozeros, Archimedean ordered, and locally compact

and shall assume $\left[{ }^{*}\right]$ for the rest of this section. In $\S 5$ we show that they are precisely the groups $\left(R^{m}, \leqq,(5)\right.$.

By $\S 2,7^{\circ}, \bar{P}$ is the positive cone of an ordering $\leqslant$. Notice that

$$
a>0, b \geqslant 0 \text { imply } a+b>0 .
$$

Let $b \in \operatorname{slb}(F)$. By $1^{\circ}$, the set

$$
Q_{b}=\left(b+P^{*}\right) \cap \operatorname{slb}(F)
$$

is non-empty and directed to the right; moreover $Q_{b} \subseteq(b, a)$ where $a$ is any element of $F$. We regard $Q_{b}$ as a net, indexing the elements by themselves; $(b, a)^{-}$ is compact by $\S 2,15^{\circ}$, so the net has an accumulation point $l$ say, in $(b, a)^{-}$. Using the fact that $Q_{b}$ is an increasing net, one shows by standard arguments that $Q_{b}$ actually converges to 1 . Since $(G, \mathfrak{u})$ is Hausdorff, $Q_{b}$ determines $l$ uniquely as its only limit point.

It is clear that $l$ does not depend upon $a$; we show that it does not depend upon the choice of $b$. Assuming for the moment the contrary, write $l_{b}$ for $l$ as defined above. First, note that

$$
b<l_{b} \text {. }
$$

For there exist $x_{1}, x_{2}$ in $Q_{b}$ such that $b<x_{1}<x_{2}$, and $e$ such that $0<e<x_{2}-$ $x_{1}$. By convergence of the net there exists $x_{3} \in Q_{b}$ for which $l_{b}-e<x_{3}<l_{b}+e$ and $x_{3}>x_{2}$; but then $x_{2}<x_{3}<l_{b}+x_{2}-x_{1}$, so $b<x_{1}<l_{b}$.

Let $c \in \mathrm{slb}(F), b<c$; we prove $l_{b}=l_{c}$. Let $e>0$ be given. By order-denseness we can write

$$
e=e_{1}+e_{2}, e_{1}>0, e_{2}>0 .
$$

Then there exists $x_{1} \in Q_{b}$ such that

$$
x>x_{1}, x \in Q_{b} \Rightarrow l_{b}-e_{1}<x<l_{b}+e_{1}
$$

and $x_{2} \in Q_{c}$ such that

$$
x>x_{2}, x \in Q_{c} \Rightarrow l_{c}-e_{2}<x<l_{c}+e_{2} .
$$

Choose some $x>x_{1}, x_{2}, c, x \in \operatorname{slb}(F) ; 1^{\circ}$ asserts that such $x$ exists. Then 
$x \in Q_{c}$, and $x \in Q_{b}$ since $c>b$. So we have

whence

$$
\begin{aligned}
& l_{b}-e_{1}<x<l_{c}+e_{2}, \\
& l_{c}-e_{2}<x<l_{b}+e_{1},
\end{aligned}
$$

$$
-e=-\left(e_{1}+e_{2}\right)<l_{b}-l_{c}<e_{1}+e_{2}=e .
$$

Since $\mathfrak{U}$ is Hausdorff, it follows that $l_{b}=l_{c}$.

Finally, if $b, b^{\prime} \in \operatorname{slb}(F)$ there exists $c \in \operatorname{slb}(F)$ with $b, b^{\prime}<c$, and $l_{b}=l_{c}=l_{b^{\prime}}$. Thus $l$ is independent of $b$ as asserted, and

$$
b<l \text {, for all } b \in \operatorname{slb}(F) .
$$

Next, we note that

$$
l \leqslant a \text {, for all } a \in F .
$$

In fact if $a \in F$ then $l \in(b, a)^{-}=\llbracket b, a \rrbracket$ by $\S 2,8^{\circ}$, so $l \leqslant a$. We have now proved a portion of

$2^{\circ}$. Given a non-empty compact subset $F$ of $G$, there exist two elements $l, u$ in $G$ satisfying

$$
\begin{aligned}
& x<l \leqslant a \text { for all } x \in \operatorname{slb}(F), a \in F, \\
& a \leqslant u<y \text { for all } a \in F, y \in \operatorname{sub}(F),
\end{aligned}
$$

and these conditions determine $l, u$ uniquely.

The statements about $u$ have proofs analogous to those about $l$; so to complete the proof it will suffice to show that (3.3) determines $l$ uniquely. Suppose $e>0$, and let $l^{\prime}$ be another element satisfying (3.3). If $a \in F$ then $a-l \in \bar{P}$ so $e+a-l>0$. Thus $l-e \in \operatorname{slb}(F)$, so $l-e<l^{\prime}$. By reversing the roles of $l$ and $l^{\prime}$ we get $-e<l-l^{\prime}<e$, for all $e>0$, and hence $l=l^{\prime}$. $/ /$

We shall write $\inf (F)$ and $\sup (F)$ for $l$ and $u$ respectively. From the definitions it follows that

$$
\sup (F)=-\inf (-F) .
$$

3. $x<\inf (F)$ if and only if $x \in \operatorname{slb}(F)$,

$y>\sup (F)$ if and only if $y \in \operatorname{sub}(F)$.

The proofs are straightforward. These properties in fact characterize inf and sup respectively. (This remark is due to N. Cameron.)

$4^{\circ}$. If $\operatorname{slb}(a)=\operatorname{slb}(b)$, then $a=b$.

Proof. Here slb $(a)$ means of course $\operatorname{slb}(\{a\})$. We are given that $x<a$ iff $x<b$. If $e>0$ then $a-e<a$ so $a-e<b, e+b-a>0$. Thus $b-a \geqslant 0$. Similarly $a-b \geqslant 0$. Therefore $a=b$. // 
Given $a, b$ in $G$, the doubleton $\{a, b\}$ is compact; define $\wedge$ and $\vee$ by

$$
a \wedge b=\inf \{a, b\}, a \vee b=\sup \{a, b\} .
$$

It was pointed out in $\S 2,9^{\circ}$ that $(G, \leqq)$ is an antilattice. By contrast, $a \wedge b$ and $a \vee b$ are always defined; we show presently that they are in fact the lattice meet and join for the ordering $\leqslant$, and that $(G, \preccurlyeq)$ is a lattice-ordered group. It is not necessary to point out that they are not the lattice operations in $(G, \leqq)$ in general.

$$
\text { 5. } \begin{aligned}
(a+c) \wedge(b+c) & =(a \wedge b)+c, \\
(a+c) \vee(b+c) & =(a \vee b)+c .
\end{aligned}
$$

Proof. This uses $3^{\circ}$ and $4^{\circ}$. For $x \in G$,

$$
\begin{aligned}
x<(a+c) \wedge(b+c) & \Leftrightarrow x<a+c \text { and } x<b+c \\
& \Leftrightarrow x-c<a \wedge b,
\end{aligned}
$$

so the first equation holds. The second is proved similarly. //

$6^{\circ}$. TheOREM. When $a, b \in G, a \wedge b$ and $a \vee b$ are the lattice meet and join of $a, b$ in $(G, \preccurlyeq)$, and $(G, \preccurlyeq)$ is a lattice-ordered group.

Proof. It has to be shown that (i) $a \wedge b \leqslant a, b$, (ii) $x \leqslant a, b \Rightarrow x \leqslant a \wedge b$, with similar statements for $\vee$. Of these, (i) follows immediately from the property (3.3). To verify (ii) let $x \leqslant a, b$, and suppose $e>0$. Then $x<a+e, b+e$, so $x<(a+e) \wedge(b+e)=a \wedge b+e$ by $5^{\circ}$, i.e. $e+(a \wedge b-x)>0$. Therefore $a \wedge b-$ $x \geqslant 0 . / /$

We could at this stage go on to show the existence of $\inf (S)$ and $\sup (S)$ for all $\sigma$-compact sets $S \subseteq G$, suitably bounded. For example, for a non-empty $\sigma$-compact set $S$ bounded below, $\inf (S)$ is the unique element $p$ satisfying $x \leqslant p \leqslant a$ for all $x \in \operatorname{slb}(S)$ and all $a \in S$. Adjunction to $G$ of two elements $-\infty$ and $\infty$ allows somewhat more general statements to be made, and provides a suitable compactification of $(G, \mathfrak{U})$. It can also be shown that $(G, \mathfrak{u})$ is complete, as a uniform space. In view of the main result $\S 5,2^{\circ}$ below, we do not pursue these matters.

\section{The lattice structure for $(G, \preccurlyeq)$}

We examine the consequences of supposing that $(G, \preccurlyeq)$ is a lattice-ordered group. By $\S 3,6^{\circ}$, this is the case in the situation [ $\left.{ }^{*}\right]$; but since it can also occur when $(G, \mathfrak{H})$ is not locally compact, we shall work generally under the weaker assumption:

$(G, \leqq, \mathfrak{U})$ is a tight Riesz group without pseudozeros, and such that $(G, \preccurlyeq)$ is a lattice-ordered group.

$1^{\circ}$. The maps $\langle a, b\rangle \mapsto a \wedge b$ and $\langle a, b\rangle \mapsto a \vee b$ from $(G \times G, \mathfrak{U} \times \mathfrak{U})$ onto $(G, \mathfrak{u})$ are continuous. 
ProOF. Let $e>0$ be given, and let $\left\{a_{\lambda}\right\}_{\lambda \in A},\left\{b_{\mu}\right\}_{\mu \in M}$ be nets converging to $a, b$ respectively. If

then

$$
-e<a-a_{\lambda}<e,-e<b-b_{\mu}<e
$$

$$
\begin{aligned}
a_{\lambda} \wedge b_{\mu}-e & =\left(a_{\lambda}-e\right) \wedge\left(b_{\mu}-e\right)<a \wedge b \\
& <\left(a_{\lambda}+e\right) \wedge\left(b_{\mu}+e\right)=a_{\lambda} \wedge b_{\mu}+e,
\end{aligned}
$$

so $-e<a \wedge b-a_{\lambda} \wedge b_{\mu}<e$. The result for $\wedge$ follows; the proof for $\vee$ is similar. //

$2^{\circ} .(G, \preccurlyeq)$ is a distributive lattice; the ordering is isolated; $G$ is torsion-free.

For proofs of these properties of lattice-ordered groups see Fuchs (1963), p. 67.

The following elementary lemma on the existence of small elements is particularly useful; it does not require the assumption at the head of this section.

$3^{\circ}$. Lemma. Let $(G, \leqq, \mathfrak{u})$ be a tight Riesz group. Given $s>0$ in $G$ and positive integer $n$, there exists $t>0$ in $G$ such that $0<n t<s$.

Proof. $(G, \mathfrak{U})$ is a topological group by $\S 2,3^{\circ}$, so given any neighbourhood $V$ of 0 there exists a base neighbourhood $W$ of 0 such that $W+W+\cdots+W \subseteq V$, for a specified number $n$ of summands. Expressing this in terms of the open orderintervals of $G$ leads to the stated result. //

$4^{\circ}$. THEOREM. The ordering $\leqq$ is isolated: in fact if $a \in G$ and $n$ is a positive integer,

$$
\begin{aligned}
& n a \succ 0 \Rightarrow a>0, \\
& n a>0 \Rightarrow a>0, \\
& n a=0 \Rightarrow a=0 .
\end{aligned}
$$

ProOF. We have already remarked upon the first and last implication. Suppose $n a>0$, so that $a>0$, and assume contrary to the theorem that $a \in \bar{P} \backslash P$. Then $a \in \partial P=\partial \bar{P}$ (by $\S 2,6^{\circ}$, (i)), so $a \in(G \backslash \bar{P})^{-}$. Let $\left\{a_{\lambda}\right\}_{\lambda \in \Lambda}$ be a net in $G \backslash \bar{P}$ converging in $\mathfrak{U}$ to $a$. We must have $n a_{\lambda} \in G \backslash \bar{P}$, for $n a_{\lambda} \in \bar{P}$ implies $a \in \bar{P}$. By continuity, $n a_{\lambda} \rightarrow n a$; so $n a \in(G \backslash \bar{P})^{-}$, which is not possible if also $n a \in P^{*}$. This contradiction concludes the proof.//

Another consequence of the lattice structure is:

$5^{\circ} .(G, \preccurlyeq)$ is a loose Riesz group.

For every lattice has the loose Riesz property: given $a_{1}, a_{2} \leqslant b_{1}, b_{2}$ we have $a_{1}, a_{2} \leqslant a_{1} \vee a_{2} \leqslant b_{1}, b_{2}$.

Not every loose Riesz group is lattice-ordered: see Fuchs (1965), §7.

$6^{\circ}$. TheOREM. $(G, \mathfrak{U})$ has no compact subgroups other than $(0)$.

Proof. Let $H$ be a compact subgroup of $G$, different from (0). Suppose that $z<2 a$ for all $z \in H, a \in P^{*}$. Then also $-2 a<z<2 a$ for all $z \in H$, 
$a \in P^{*}$; and from $3^{\circ}$ it follows that $-b<z<b$ for all $z \in H, b \in P^{*}$, which is impossible since $H \neq(0)$.

Thus there exists a pair of elements $z \in H, a \in P^{*}$ such that $z \nless 2 a$. Then $z \neq 0$. Also $n z \nless 2 a$ for positive $n \geqq 2$; for if $n z<2 a$ then $n z<n a$, so $z<a<2 a$ by $4^{\circ}$.

Using this $a$, we argue as in the proof of $\S 2,12^{\circ}$, to show the existence of elements $t_{1}, t_{2}, \cdots, t_{n}$ in $H$ such that, for every $x \in H$ there exists some integer $j_{x}, 1 \leqq j_{x} \leqq n$, such that $x-a<t_{j_{x}}<x+a$. Choose for $x$ first any element $x_{0} \in H$, and subsequently the elements

$$
x_{k}=x_{0}+k z \text { for } k=0,1,2, \cdots .
$$

The intervals $\left(x_{k}-a, x_{k}+a\right)$ are all pairwise disjoint. For if $y \in\left(x_{k_{1}}-a, x_{k_{1}}+a\right) \cap$ $\left(x_{k_{2}}-a, x_{k_{2}}+a\right)$ with $k_{1}<k_{2}$, then $x_{k_{2}}-a<y<x_{k_{1}}+a$, so

$$
\left(k_{2}-k_{1}\right) z=x_{k_{2}}-x_{k_{1}}<2 a,
$$

which we have shown is impossible. As in $\S 2,12^{\circ}$, the disjointness of the intervals implies a contradiction. //

$7^{\circ}$. If, in addition to the other assumptions, $(G, \leqq)$ is Archimedean ordered, then $(G, \mathfrak{u})$ is first countable.

Proof. Take any $s \in P^{*}$. We can by using $3^{\circ}$ construct inductively a sequence $\left\{t_{n}\right\}_{n=0}^{\infty}$ for which $t_{0}=s$ and

$$
0<n t_{n}<s, n t_{n-1}, \quad 0<t_{n}, \quad \text { for } n=1,2, \cdots .
$$

Let $(-e, e)$ be any base neighbourhood of 0 , so that $e>0$. By the Archimedean property there exists $n$ such that $n e>s$, and so $n e>n t_{n}$. By $4^{\circ}$ this implies $e>t_{n}$. We also have $t_{n}<t_{n-1}$. Thus $\left(-t_{n}, t_{n}\right), n=1,2, \cdots$, is a countable base for $\mathfrak{U}$ at 0 , in fact a decreasing sequence of neighbourhoods. //

Note that the lattice property of $\leqslant$ is not used directly in the proofs of $6^{\circ}$ and $7^{\circ}$; it is enough to know that $\leqq$ is isolated.

The absence of non-trivial compact subgroups has the important consequences that $G$ is radical-free and that the real characters on $G$ are point-separating. The property is used in the next section.

\section{Consequences of the condition [*]}

In this final section we prove the result promised above, namely that a group satisfying $\left[{ }^{*}\right]$ is isomorphic to $\left(R^{m}, \leqq, \mathfrak{U}\right), m$ some positive integer.

$1^{\circ}$. If $(G, \leqq, \mathfrak{U})$ satisfies $\left[^{*}\right]$ then $(G, \mathfrak{U})$ is algebraically and topologically isomorphic to $\left(R^{m}, \mathbb{E}\right)$ for some positive integer $m$, \& being the Euclidean topology.

Proof. By $\S 4,6^{\circ} G$ is radical-free, and so by a result of W. Mackey (1948) 
(or see Hewitt and Ross (1963), p. 390) there is an algebraic and topological isomorphism $\phi$ of $G$ onto $R^{m} \oplus D$ for some positive integer $m$ and some discrete group $D$. Let $\xi \in R^{m}$, with $\xi>0$ in the ordering defined by (1.1), so that $(-\xi, \xi)$ is open in $\left(R^{m}, \mathbb{E}\right)$ and $(-\xi, \xi) \oplus\{0\}$ is open in $\phi(G)$. Thus there exists $a \in P^{*}$ with

$$
\phi((0, a)) \subseteq \phi((-a, a)) \subseteq(-\xi, \xi) \oplus\{0\} .
$$

But then by $\S 2,4^{\circ}$ III,

$$
\begin{aligned}
\phi((0, n a)) & =\phi((0, a))+\phi((0, a))+\cdots+\phi((0, a)) \\
& \subseteq(-n \xi, n \xi) \oplus\{0\} \subseteq R^{m} \oplus\{0\}
\end{aligned}
$$

for each positive integer $n$. Since $P^{*}=\bigcup_{n}(0, n a)$ and $G=P^{*}-P^{*}$ it follows that $\phi(G)=\phi\left(P^{*}\right)-\phi\left(P^{*}\right) \subseteq R^{m} \oplus\{0\}$, and hence $D$ must be trivial. //

Using the map $\phi$ we may define an order $\mathcal{E}$ on $R^{m}$ by

$$
x=\phi(a) \succ 0 \text { if and only if } a \geqslant 0 \text { in } G .
$$

With this ordering $R^{m}$ becomes a lattice-ordered group and, indeed, a real vector lattice. For if $x \in R^{m}$ and $x<0$ then $n x<0$ certainly, and $x / n<0$ using $\S 4,2^{\circ}$, for each positive integer $n$, and so $\alpha x \prec 0$ for rational $\alpha>0$. Since $\phi(\bar{P})$ is closed it follows that $\alpha x<0$ for all real $\alpha>0$. But then by the Choquet-Kendall theorem (Peressini $(1967)$, pp. 9, 10) there are vectors $v^{(k)}, k=1,2, \cdots, m$, spanning $R^{m}$, such that ${ }^{2}$

$$
x \prec 0 \text { if and only if }\left\langle x, v^{(k)}\right\rangle \geqq 0 \text { for } k=1,2, \cdots, m .
$$

These $v$ 's are not in general the extreme rays of the positive cone for $\zeta$ in $R^{m}$. Setting $S=\left(\left\langle v^{(i)}, v^{(j)}\right\rangle\right), \quad T=S^{-1}, \quad T=\left(\tau_{i j}\right), \quad$ let $u^{(k)}=\sum_{j} \tau_{k j} v^{(j)}$. Then $\left\langle\sum_{k} \mu_{k} u^{(k)}, v^{(j)}\right\rangle=\mu_{j}$, so that

$$
x=\sum \mu_{k} u^{(k)} \succ 0 \text { if and only if } \mu_{k} \geqq 0 \text { for } k=1,2, \cdots, m .
$$

Thus if $e^{(k)}=(0,0, \cdots, 1, \cdots, 0)$, the 1 in the $k^{\text {th }}$ position, the map

$$
\theta: \sum \mu_{k} u^{(k)} \mapsto \sum \mu_{k} e^{(k)}
$$

is a lattice, algebraic and topological isomorphism of $\left(R^{m}, \preccurlyeq, \mathfrak{E}\right)$ with $\left(R^{m}, \leqslant,(\mathfrak{E})\right.$. Thus there is an isomorphism $\psi$ of $(G, \leqslant, \mathfrak{H})$ with $\left(R^{m}, \leqslant, \mathbb{E}\right)$, namely $\psi=\theta \circ \phi$.

Alternatively, we can obtain this result as follows. Let $a \in G, a>0$, and set $x=\phi(a)$. The functional $\|\cdot\|_{x}$ defined by

$$
\|y\|_{x}=\inf \{\lambda>0:-\lambda x \preccurlyeq y \preccurlyeq \lambda x\}
$$

is easily seen to be a norm on $R^{m}$, necessarily defining the topology $\&$, and under this norm $\left(R^{m}, \zeta\right)$ is an $(M)$-space with unity $x$ (see G. Birkhoff (1967), p. 376). Thus by the representation theorem of S. Kakutani, $\left(R^{m}, \zeta,(\tilde{E})\right.$ is lattice, alge-

$2\langle\cdot, \cdot\rangle$ now denotes the inner product in $R^{m}$. 
braically and topologically isomorphic with $C(X)$ for some compact space $X$. It is immediate that $X$ consists of precisely $m$ points, with discrete topology, and so $C(X)$ can be identified with $\left(R^{m}, \leqslant,[)\right.$, giving the required result.

Finally we note that using $\S 2,6^{\circ},(\bar{P})^{\circ}=P^{*}$, and so the isomorphism $\psi$ maps $P^{*}$ onto the positive cone in $\left(R^{m}, \leqq\right)$. Thus we obtain:

$2^{\circ}$. Theorem. If $(G, \leqq, \mathfrak{U})$ is an Archimedean ordered tight Riesz group without pseudozeros, and locally compact, then it is isomorphic in all respects to $\left(R^{m}, \leqq,(\mathbb{E})\right.$ for some positive integer $m$.

\section{Some examples}

Besides the group $\left(R^{m}, \leqq,(5)\right.$ already discussed, we mention the following.

$1^{\circ}$. Let $F$ be the set of all real polynomials on a compact interval in $R$, say $[0,1]$, and write $f>0$ to mean $f(t)>0$ for $0 \leqq t \leqq 1 . F$ is a partially ordered group. Its pseudopositives are the non-zeto polynomials $g$ for which $g(t) \geqq 0$ for $0 \leqq t \leqq 1$ and $g\left(t_{0}\right)=0$ for at least one $t_{0}$; so $F$ has no pseudozeros. $F$ is a tight Riesz group; for if $f_{1}, f_{2}<g_{1}, g_{2}$ then in $C[0,1]$ we have $f_{1} \vee f_{2}<g_{1} \wedge g_{2}$ and a polynomial can be interpolated strictly between these two functions. Also $F$ is Archimedean ordered. It is clear that $(F, \leqslant)$ is not lattice-ordered; it can be shown that $(F, \leqslant)$ is a loose Riesz group. $F$ is isolated, for both orderings.

$2^{\circ}$. A similar example uses the $k$-times continuously differentiable functions on $[0,1]$ in place of the polynomials.

Examples $1^{\circ}$ and $2^{\circ}$ are adapted from L. Fuchs (1966), $\S 5$.

$3^{\circ}$. Let $(G, \leqq, \mathfrak{l})$ be a tight Riesz group without pseudozeros, Archimedean ordered, and such that $(G, \preccurlyeq)$ is lattice-ordered. Let $T$ be a compact Hausdorff space, and write $\mathbb{C}$ for the additive group of all continuous functions $f: T \rightarrow G$. Ordered by taking as strict positive cone $\mathfrak{B}^{*}=\left\{f: f(t) \in P^{*}\right.$ for all $\left.t \in T\right\}$, $\mathfrak{C}$ becomes a group having the properties listed above for $G$. Its open-interval topology is the topology of uniform convergence on $T$. $(\mathbb{E}, \preccurlyeq)$ is lattice-ordered, and is therefore a loose Riesz group and isolated with respect to both orderings. The example is discussed in Miller (1972).

$4^{\circ}$. Let $(G, \leqq, \mathfrak{H})$ be a tight Riesz group, and $M$ an arbitrary abelian group. Any group homomorphism $\kappa: M \rightarrow G$ of $M$ into $G$ can be used to define a partial ordering $\leqq$ on $M$ by

$$
x>0 \text { in } M \text { if and only if } \kappa(x)>0 \text { in } G .
$$

Suppose that $\kappa(M)$ is topologically (equivalently, orderwise) dense in $G$. Then: (i) $(M, \leqq)$ is a tight Riesz group; (ii) The pseudopositives of $M$ are the set $\{x \neq 0: \kappa(x)$ is pseudopositive or zero $\}$; the pseudozeros of $M$ are the set $\{x \neq 0$ : 
$\kappa(x)$ is pseudozero or zero ; (iii) $(M, \leqq)$ is Archimedean ordered if and only if $(G, \leqq)$ is Archimedean ordered; (iv) $(M, \leqq)$ is isolated if $(G, \leqq)$ is isolated; (v) When $M$ has the open-interval topology, $\kappa$ is a continuous map.

\section{References}

G. Birkhoff (1967): Lattice theory (Amer. Math. Soc., Providence, 3rd ed.).

J. Dugundji (1966): Topology (Allyn and Bacon, Boston).

L. Fuchs (1963): Partially ordered algebraic systems (Pergamon, Oxford).

L. Fuchs (1965): Riesz groups, Annali della Scuola Normale Superiore di Pisa, Serie III, 19, 1-34.

L. Fuchs (1966): Riesz vector spaces and Riesz algebras (Queen's papers in pure and applied mathematics, Queen's University, Kingston).

E. Hewitt and K. A. Ross (1963): Abstract harmonic analysis, I (Springer, Berlin).

G. W. Mackey (1948): The Laplace transform for locally compact abelian groups, Proc. Nat. Acad. Sci. U.S.A. 34, 156-162.

J. B. Miller (1970): Higher derivations on Banach algebras, Amer. J. of Math. 92, 301-331.

J. B. Miller (1972): Tight Riesz groups and the Stone-Weierstrass theorem, J. Aust. Math. Soc. (to appear).

A. L. Peressini (1967): Ordered topological vector spaces (Harper and Row, New York).

F. Riesz (1940): Sur quelques notions fondamentales dans la théorie générale des opérations linéaires, Annals Math. 41, 174-206.

Carleton University

Ottawa

Trent University

Peterborough 